\title{
Development of Rapid Pharmacogenomic Testing Assay in a Mobile Molecular Biology Laboratory (2MoBiL)
}

\author{
Georgios Psarias, ${ }^{1, \star}$ Evanthia Iliopoulou,, ${ }^{1, *}$ Ioannis Liopetas, ${ }^{1}$ Anna Tsironi, ${ }^{1}$ Dimitrios Spanos, ${ }^{1}$ \\ Athina Tsikrika, ${ }^{1}$ Konstantinos Kalafatis, ${ }^{1}$ Dimitra Tarousi, ${ }^{1}$ Georgios Varitis, ${ }^{1}$ Maria Koromina, \\ Stavroula Siamoglou, ${ }^{1}$ and George P. Patrinos ${ }^{1-3}$
}

\begin{abstract}
Pharmacogenomics is rapidly assuming an integral part in modern health care. Still, its broad applicability relies on the feasibility of performing pharmacogenomic testing in all clinical settings, including in remote areas or resourcelimited settings with budget restrictions. In this study, we describe the development and feasibility of rapid and reliable pharmacogenomics assays using a portable molecular biology laboratory, namely the 2MoBiL (Mobile Molecular Biology Laboratory). More precisely, we demonstrate that the genotyping of rs4149056, located within $S L C O 1 B 1$, can be efficiently and reliably performed using the $2 \mathrm{MoBiL}$ portable laboratory and conventional benchtop laboratory equipment and a gold standard genotyping method (KASP assay) as directly comparable methodologies. Taking into account the compact size of $2 \mathrm{MoBiL}$, which directly and positively impacts on its portability, and the high accuracy achieved, we conclude that the 2MoBiL-based genotyping method is warranted for further studies in clinical practices at remote areas and resource-limited as well as time-constrained planetary health settings. To contextualize the broader and potential future applications of $2 \mathrm{MoBiL}$, we emphasize that genotyping of a limited set of clinically relevant single-nucleotide polymorphisms is often a common endpoint of genomics and pharmacogenomics discovery and translational research pipeline. Hence, rapid genotyping by $2 \mathrm{MoBiL}$ can be an essential catalyst for global implementation of pharmacogenomics and personalized medicine in the clinic. The Clinical Trial Registration number is NCT03093818.
\end{abstract}

Keywords: pharmacogenomics, genotyping, SLCO1B1, portable molecular biology laboratory, mobile molecular biology laboratory, 2MoBiL

\section{Introduction}

$\mathbf{P}$ HARMACOGENOMICS AIMS TO DETERMINE the differential drug response rates and development of adverse drug reactions (ADRs), owing to differences in patients' genomic profiles (Pirmohamed, 2001), which can subsequently positively impact on optimizing drug treatment modalities. Implementation of pharmacogenomics in the clinic is considered to be the cornerstone of personalized medicine by introducing genomics interventions as a new concept for health care systems.

To date, genetic tests are primarily used for the diagnosis of certain, mostly monogenic, diseases, such as Huntington's disease, thalassemias, and cystic fibrosis, among others.
Nevertheless, in the postgenomics era, many health care providers began using pharmacogenomics tests to adjust drug dose, and consequently, there are guidelines for genomeguided drug dose adjustment for more than 150 drugs and 750 label annotations in the Pharmacogenomics Knowledgebase (PharmGKB; www.pharmgkb.org) (Thorn et al., 2013), while similar observations about the existence of pharmacogenomics guidelines are applicable in both the United States Food and Drug Administration (www.fda.gov) and the European Medicines Agency (www.ema.europa.eu) (Koutsilieri et al., 2020).

Statins are among the drug labels that include guidance for genome-informed drug prescription, a commonly prescribed drug for hypercholesterolemia. More precisely, according to

\footnotetext{
${ }^{1}$ Laboratory of Pharmacogenomics and Individualized Therapy, Department of Pharmacy, School of Health Sciences, University of Patras, Patras, Greece.

${ }^{2}$ Department of Pathology, College of Medicine and Health Sciences, United Arab Emirates University, Al-Ain, United Arab Emirates.

${ }^{3}$ Zayed Center of Health Sciences, United Arab Emirates University, Al-Ain, United Arab Emirates.

*These two authors contributed equally to this work.
} 
the present pharmacogenomics guidelines, administration of simvastatin in patients carrying rs4149056 variant within the SLCO1B1 should be avoided, while it is also reported that the metabolism of rosuvastatin and fluvastatin is affected, although at a lower degree, by the same variant (Brunham et al., 2018; Khine et al., 2016; Stroes et al., 2015; Wilke et al., 2012).

According to a genome-wide association study, the SLCO1B1 rs4149056 was associated with an odds ratio for myopathy of 4.5 per copy of the $C$ allele and 16.9 among $\mathrm{C} / \mathrm{C}$ homozygotes compared with the T/T genotype (Meade et al., 2008). The frequencies of $S L C O 1 B 1$ polymorphisms show a significant variety among different world populations, indicating the need of pharmacogenetic testing for rs4149056 because the genomic profile for each individual is extremely dependent on population genetic architecture. Of note, no other variant known to date has been associated with statininduced myopathy, which is one of the main side effects of statin treatment (Meade et al., 2008).

Although genome-wide studies and markers continue to be relevant and of great importance, it is not uncommon to subset and select few clinically relevant biomarkers for clinical application. Herein, we selected the SLCO1B1 rs4149056 functional polymorphism (c.T521C and p.V174A) that leads to higher statin circulating concentration (Pasanen et al., 2006, 2007). The rs4149056 is a missense variant (T/C, with the ancestral allele T) located in exon 5 of SLCO1B1 within chromosome 12. Regarding rs4149056 polymorphism, the $\mathrm{C}$ allele frequency found in Caucasian descent is 0.146 and in European population 0.161; therefore, rs4149056 is a variant that occurs with great frequency among the population worldwide. Moreover, the frequencies of T/T, T/C, and C/C in the Europeans are 0.698, 0.282, and 0.020 , respectively (1000 Genomes Project).

Taking the above into consideration, in this study, we demonstrate the feasibility of using a fully equipped portable molecular biology laboratory (referred to as "2MoBiL" method) as a quick and straightforward method for rs4149056 pharmacogenomics testing. This portable laboratory has been already successfully used for educational purposes in high school and college students all over Greece and abroad (Siamoglou and Patrinos, 2019), yielding comparable genotyping results compared with conventional laboratory methods and instrumentation.

\section{Materials and Methods}

\section{Samples, deoxyribonucleic acid extraction, and genotyping}

Eighty-one volunteers of Greek origin were recruited for genotyping, who were diagnosed with a variety of neuropsychiatric disease phenotypes. Written informed consent was obtained from all participating patients and the study was approved by the Institutional Ethics Committee.

Genomic deoxyribonucleic acid (DNA) was extracted from peripheral blood using a DNA extraction Kit (NucleoSpin Blood; Macherey-Nagel). All samples were genotyped using the KASP assay, using the Hydrocycler ${ }^{-4}$ (LGC Genomics) coupled to FLUOstar Omega SNP (BMG LABTECH), as the gold standard genotyping method, which was standardized against Sanger sequencing methodology. The method utilizes two fluorescently labeled oligonucleotides that consist of a specific $3^{\prime}$ primer sequence region and a $5^{\prime}$ hairpin region that is labeled with either of two fluorophores. The presence of the $3^{\prime}$ primer sequence enables these fluorescent oligonucleotides to work with target-specific unlabeled primers designed to the researcher's specific target sequence. Oligonucleotide sequences and amplification conditions are available upon request (https://www.bmglabtech.com).

DNA genotyping was performed for the aforementioned samples and more specifically for rs4149056 within SLCO1B1. Amplification was conducted according to FirePol Taq protocol (Solis Biodyne) (method details available upon request). Regarding the rs4149056, an allele-specific polymerase chain reaction (AS-PCR) method was standardized with two alternative forward primers hybridizing exclusively either to the $\mathrm{T}$ or $\mathrm{C}$ allele, respectively.

\section{Statistical analysis}

To further validate our in vitro findings, a comparison between the results from the 2MoBiL (Mobile Molecular Biology Laboratory) setting and those that came from a conventional benchtop laboratory equipment was conducted, while using as a gold standard the results produced from the KASP assay (Fig. 1). The comparison was based on genotype and score predictions, both derived for each sample and for each method, which were assigned by 11 independent qualified evaluators. The evaluators were asked to identify the genotype and rate each sample ( $N=81$ samples) from a scale of 1 to 4 (Table 1), depending on the occurrence of the respective band within all the agarose gels produced. To further underscore the strength of the study design, the evaluators comparing the two methods were blind to the methodology involved (i.e., double-blind assessors).

Moreover, the median value was calculated for each sample to give a representative value of all 11 independent observations. To allow a comparison between the two methods, we implemented the Fisher's test by creating a $2 \times 2$ contingency table in the GraphPad software and calculating the corresponding $p$-value. To further estimate the validity of the $2 \mathrm{MoBiL}$ method and the conventional genotyping approach, 10 randomly selected samples were tested 7 times using either the $2 \mathrm{MoBiL}$ method or the conventional benchtop laboratory method. The graphical representation of the comparison between the $2 \mathrm{MoBiL}$ and the conventional genotyping approach as well as a graph depicting the validity and reproducibility of the $2 \mathrm{MoBiL}$ were created using Jupyter Notebook and the programming language, Python 3, including the libraries Seaborn (version 0.10.1) and Pandas (version 1.0.3).

\section{Results}

\section{Comparison of the two genotyping methods}

$2 \mathrm{MoBiL}$ versus conventional benchtop laboratory instrument. Overall, we performed genotyping analysis for 81 samples using both methods, the conventional one and the $2 \mathrm{MoBiL}$, based on the same principle. Herein, we present the results for an indicative number of these samples. More precisely, Table 2 depicts the genotypes of four samples as derived from the gold standard method and the performance of the other two aforementioned methods (the conventional benchtop laboratory equipment and the $2 \mathrm{MoBiL}$ ), which produced highly accurate results. Moreover, as shown in 


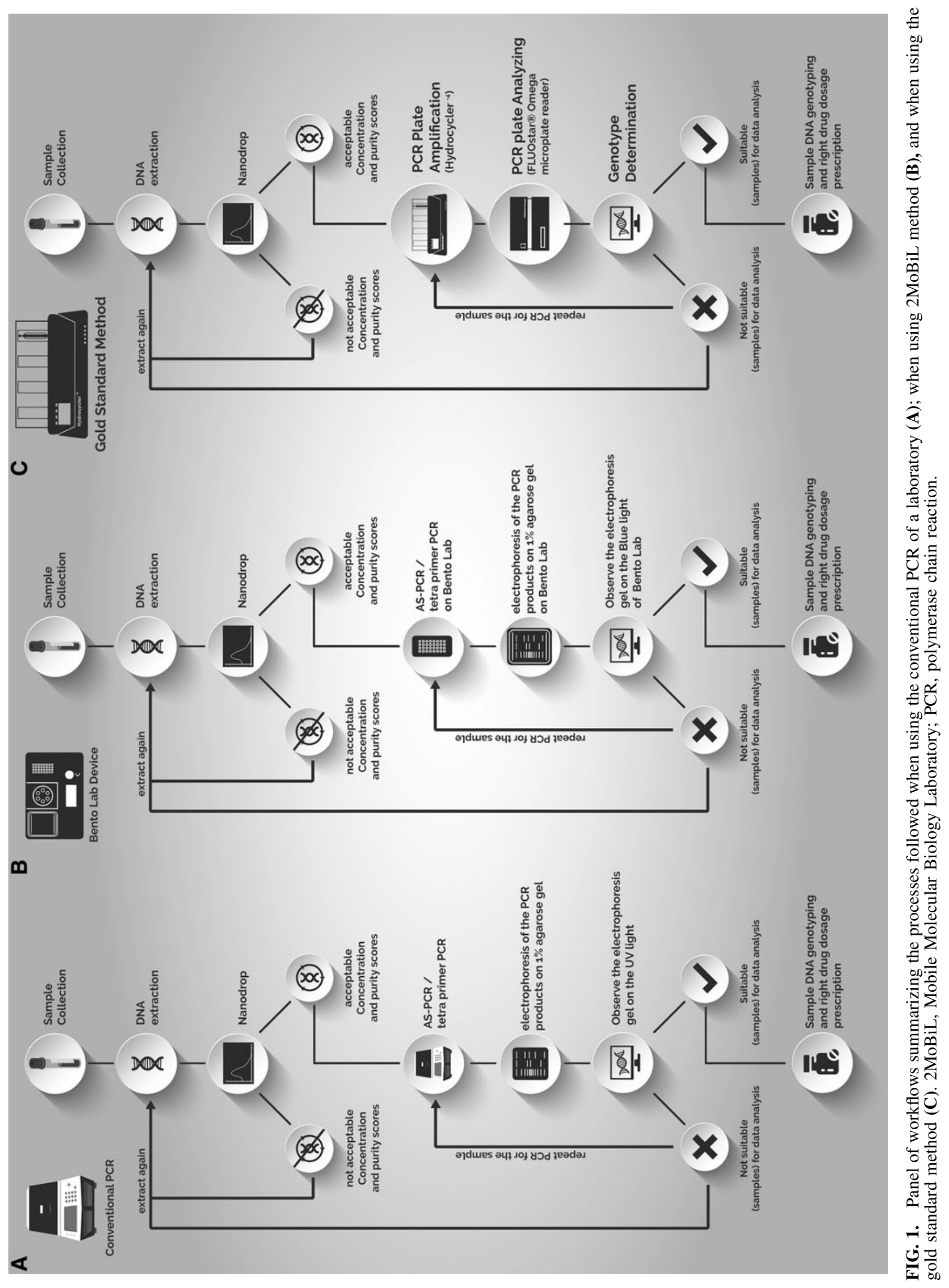


Table 1. Description of the Respective Scores Used in Both THE Conventional AND THE 2MoBiL METHOD

Score
"1" $\begin{gathered}\text { Description } \\ \text { incorrect compared to the gold standard method } \\ \text { and needs to be re-examined }\end{gathered}$
"2" $\begin{aligned} & \text { Samples with a dubious genotype } \\ & \text { "3" }\end{aligned}$
$\begin{aligned} & \text { Samples with a correct genotype, which could be } \\ & \text { accompanied by a faint nonspecific product, that } \\ & \text { is, a faint band of DNA on the well of mutant } \\ & \text { allele in a sample T/T and a faint band on the well } \\ & \text { of the wild-type allele in a sample C/C } \\ & \text { Samples where the correct genotype is easily } \\ & \text { detected, and the error probability is reduced }\end{aligned}$
"4"

2MoBiL, Mobile Molecular Biology Laboratory; DNA, deoxyribonucleic acid.

Figure 2, we also present the results from AS-PCR as performed for four samples and by following the protocols for the gold standard method, the conventional benchtop laboratory method, and the $2 \mathrm{MoBiL}$ method.

Regarding the scoring assessment (see Materials and Methods section; Table 1) of the 81 samples, percentages of concordance between the 3 methods were also estimated. When it comes to the conventional benchtop laboratory method, 77 out of 81 samples (95\%) were correctly identified according to the gold standard method (i.e., for samples with scores 3 and 4). Regarding the 2MoBiL method, 94\% (76 out of 81samples) of concordance against the gold standard method was observed for samples with scores 3 and 4, which were the samples with a genotype that can be used for further statistical assessment.

\section{Statistical evaluation}

The same samples were tested in both methods. To assess the significance of differences between the two genotyping methods, a Fisher's exact test was conducted. As described above, scores 1 and 2 corresponded to the FALSE values, whereas scores 3 and 4 scores corresponded to the TRUE values. We hypothesized that the genotyping ability of $2 \mathrm{Mo}-$ $\mathrm{BiL}$ and the conventional benchtop method is no different, as expected, since both were thermal cyclers based on the Peltier

Table 2. Representation of the Genotypes of Four Different Deoxyribonucleic ACid Samples (A, B, C, D) FOR RS4149056, ACCORDING TO THE GOLd STANDARD Method, The Conventional Benchtop Laboratory EQUIPMENT, AND THE 2MoBiL METHOD

\begin{tabular}{|c|c|c|c|c|c|}
\hline $\begin{array}{l}\text { Sample } \\
\text { ID }\end{array}$ & $\begin{array}{l}\text { Ref. } \\
\text { allele }\end{array}$ & $\begin{array}{l}\text { Alt. } \\
\text { allele }\end{array}$ & $\begin{array}{l}\text { GT in } \\
\text { gold } \\
\text { standard } \\
\text { method }\end{array}$ & $\begin{array}{l}\text { GT in } \\
\text { conventional } \\
\text { laboratory } \\
\text { equipment }\end{array}$ & $\begin{array}{c}G T \text { in } \\
2 M o B i L\end{array}$ \\
\hline "A" & $\mathrm{T}$ & $\mathrm{C}$ & $\mathrm{T} \mid \mathrm{T}$ & $\mathrm{T} \mid \mathrm{T}$ & $\mathrm{T} \mid \mathrm{T}$ \\
\hline "B" & $\mathrm{T}$ & C & $\mathrm{T} \mid \mathrm{C}$ & $\mathrm{T} \mid \mathrm{C}$ & $\mathrm{T} \mid \mathrm{C}$ \\
\hline "C" & $\mathrm{T}$ & $\mathrm{C}$ & $\mathrm{C} \mid \mathrm{C}$ & $\mathrm{C} \mid \mathrm{C}$ & $\mathrm{C} \mid \mathrm{C}$ \\
\hline "D" & $\mathrm{T}$ & $\mathrm{C}$ & $\mathrm{T} \mid \mathrm{T}$ & $\mathrm{T} \mid \mathrm{T}$ & $\mathrm{T} \mid \mathrm{T}$ \\
\hline
\end{tabular}

Alt. allele for the alternative one; GT indicates the genotype; Ref. allele stands for the reference allele. effect. The $p$-value was higher than the 0.05 , so our null hypothesis is not being rejected, and the association between the two examined methods is not statistically significant.

Therefore, our proposed 2MoBiL-based method can successfully produce results comparable with a benchtop laboratory method and the gold standard, while future studies in larger samples are warranted. The comparison between the $2 \mathrm{MoBiL}$ and the benchtop laboratory equipment is represented in Figure 3.

To further assess the $2 \mathrm{MoBiL}$ method in predicting the correct genotype, 10 randomly selected samples were re-run 7 times each. As shown in Figure 4, none of 10 samples deviated from the standard flat joint line. This study provides evidence that the $2 \mathrm{MoBiL}$ is as accurate as any conventional benchtop laboratory method, while also allowing the identification of the correct genotype in a high percentage of samples and repeats. Consequently, the $2 \mathrm{MoBiL}$ method is proposed as a valid and reliable method for genotyping in clinical practice for further consideration and evaluation in planetary health settings.

\section{Discussion}

Transportable molecular biology laboratories can be classified into two main categories, either mobile or portable, while taking into account their characteristics (i.e., size). In a mobile laboratory, the rear part of a vehicle is used, such as a truck, van, or even a bus, where the appropriate equipment is placed. In contrast, a portable laboratory is defined as a set of instruments that can be carried alongside, such as a centrifugal device or a conventional thermal cycler (Zaky et al., 2018). A characteristic example of a mobile laboratory is the BioBus, which is a novel concept in science education involving a bus that has been equipped as a self-contained, laboratory used to educate students in biology basics.

In contrast, a portable laboratory is much smaller in size, usually in the size of a suitcase or a smartphone (Ahrberg et al., 2016). Experimental procedures in these types of laboratories are much faster (Marx, 2015), while such laboratories are either battery operated (Nair et al., 2016) or supplied by an external power source from a nearby vehicle, thus addressing the power source issue.

One of the most characteristic examples of portable laboratories' applications is toward the protective biodefense purposes, not to mention infectious outbreaks. Over the last decades, many techniques and devices have been developed for the detection of HIV (Jangam et al., 2013), Ebola virus (Kurosaki et al., 2016), and influenza A (H1N1) virus (Lim et al., 2014), and the early diagnosis of tropical diseases such as malaria (Nair et al., 2016; Visser et al., 2015). Implementation of portable molecular biology laboratories has been also expanded to molecular diagnostics.

The ability and accuracy in detecting genomic variants by using these devices have contributed to the diagnosis of hemochromatosis disease (Macleod et al., 2016) and myeloproliferative neoplasms by detecting the human p.C282Y hemochromatosis variant using the electrochemical microarray method and $J A K 2 \mathrm{p} . \mathrm{V} 617 \mathrm{~F}$ and $M P L \mathrm{p} . \mathrm{W} 515 \mathrm{~K} / \mathrm{L}$ variants using the loop-mediated isothermal amplification technique, respectively (Wang et al., 2017).

In this study, we showed that portable laboratories and more precisely the $2 \mathrm{MoBiL}$ method can be used as a reliable, 
A

\section{$100 \mathrm{bp}$}

Ladder Aw Am Bw Bm Cw Cm Dw Dm

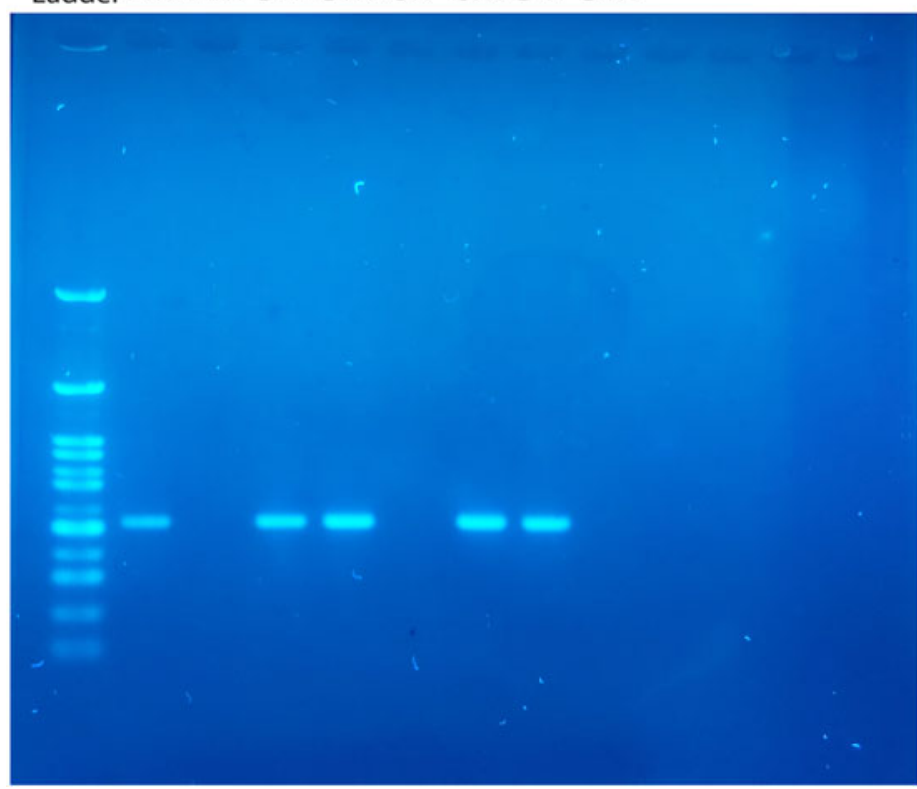

B

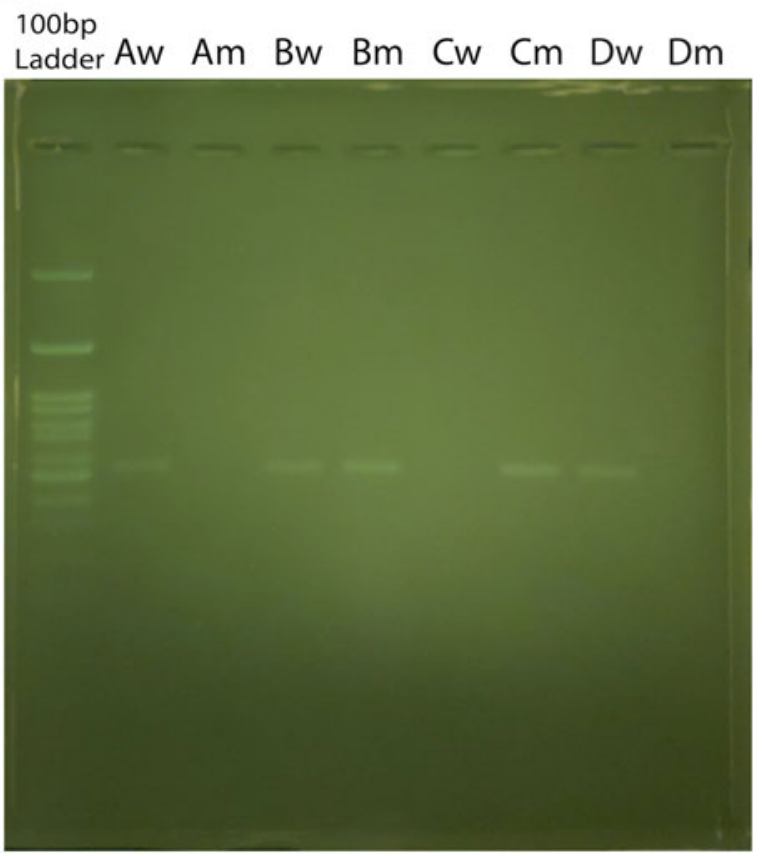

FIG. 2. Representation of the rs4149056 genotype for four different DNA samples (A, B, C, and D), as also described in Table 2, by using (A) the static laboratory equipment and (B) the 2MoBiL method. For each of the DNA samples, two wells match: for the wild type ("w," referring to T allele) and for the mutant allele ("m," referring to C allele). Ladder $100 \mathrm{bp}$ was used (Nippon Genetics) and the expected molecular weight per PCR product is 535 bp. DNA, deoxyribonucleic acid.

diagnostic approach for individualizing statin as a case study (Teama, 2018; Tuteja and Rader, 2018; Wilke et al., 2012). We emphasize that the 2MoBiL-based genotyping method has many significant advantages, in comparison to currently used rs4149056 genotyping methods, using benchtop equipment.

We note that 81 samples were selected and genotyped, using the $2 \mathrm{MoBiL}$ method and the conventional laboratory equip-

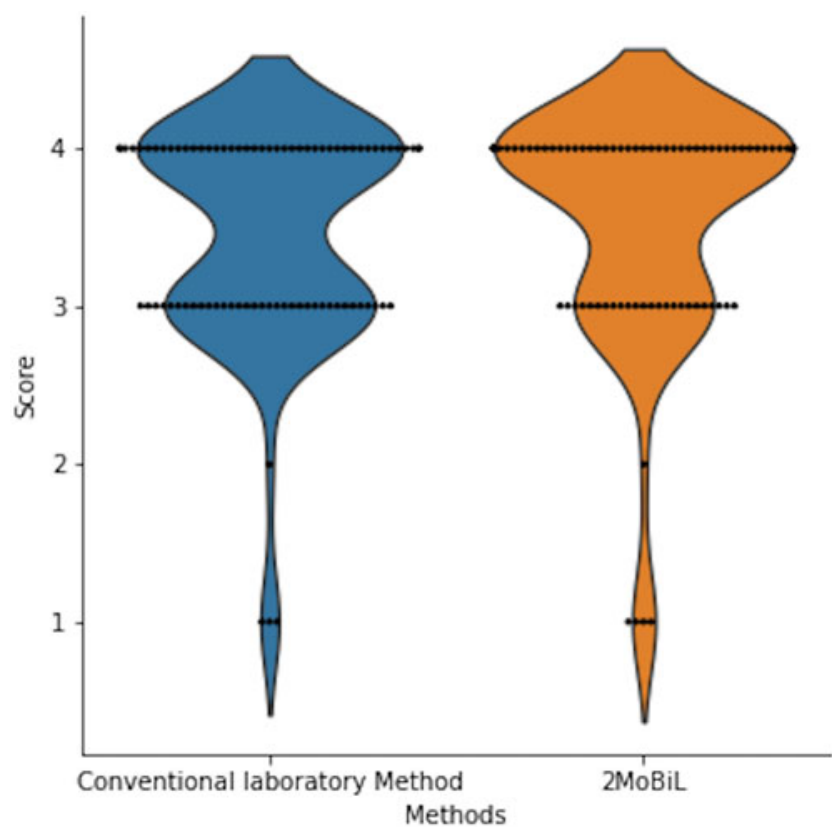

FIG. 3. Comparison between the $2 \mathrm{MoBiL}$ method and the conventional method according to the median scores for the 81 samples, as derived from the assigned quality scores. ment, and were subsequently compared and evaluated for their accuracy against the gold standard method. Both methods led to results comparable to the gold standard. More precisely, the 2MoBiL-based method successfully performs an accurate genotype analysis (94\%), providing results comparable with

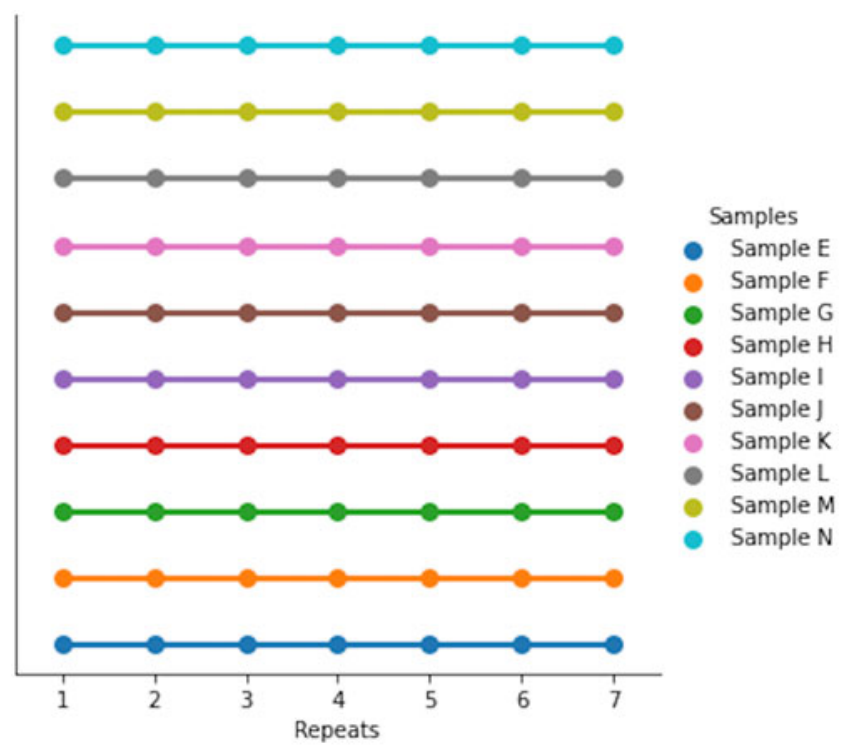

FIG. 4. Repeats for the $2 \mathrm{MoBiL}$ method after running AS-PCR for 10 different samples 7 times each. Each one of the 7 times run per sample was a complete AS-PCR assay conducted. The spots on the standard flat joint line represent the samples with the correct genotype. The dots that deviate from the standard flat joint line represent samples that gave wrong results when repeating the experiment. AS-PCR, allele-specific polymerase chain reaction. 
the conventional benchtop laboratory equipment (95\%) and the gold standard method. It is important to highlight the fact that both methods achieved a high percentage of concordance against the gold standard method, thus pointing out the accuracy and reliability of both methods.

However, any minor deviation from the gold standard method can be explained by random experimental factors, such as the nucleic acid stain used in the preparation of the agarose gel, which can often lead to misinterpretation of the predicted genotypes by the qualified evaluators. Moreover, the compared methods were tested for their validity and reliability. To this end, five randomly selected samples were tested using both methods under the same conditions. The results highlighted the greater performance of the $2 \mathrm{MoBiL}$, indicating that the latter method can be considered an accurate and reliable approach for further evaluation toward pharmacogenomic testing in clinical practice, especially in remote areas and resource-limited settings.

One of the advantages of the $2 \mathrm{MoBiL}$ laboratory is its potentially lower operational cost compared to the cost of Sanger sequencing or to the cost of the KASP assay, mainly due to the fact that the $2 \mathrm{MoBiL}$ laboratory is an all-in-one device, including the thermal cycler, the centrifuge, and the gel electrophoresis tank and transilluminator, as opposed to the KASP assay and the Sanger sequencing, both requiring a much more sophisticated and expensive benchtop equipment (Siamoglou et al., 2020).

Second, genotyping with the 2MoBiL-based method compared to the conventional one is much faster, thus reducing the waiting time to generate the result, which can be rather crucial when individualizing the drug dose. To add to this, the estimated budget to maintain a fully equipped benchtop laboratory equipment is excessively high, while this cost is poised to be significantly reduced with the 2MoBiL-based method. Studies of cost-effectiveness and utility analyses in large patient samples are warranted in the future in diverse planetary health settings and applications.

We show herein that the $2 \mathrm{MoBiL}$ method can be used as a reliable, diagnostic approach for individualizing statin administration with substantial alterations in the risk of simvastatin-induced myopathy. These findings offer prospects for applications to other diagnostic approaches for individualizing other drugs with pharmacogenomics importance, such as chloroquine and rasburicase. According to PharmGKB, prescribing chloroquine or rasburicase without considering the potential G6PD (glucose-6-phosphate dehydrogenase) genetic variation can lead to severe ADRs such as hemolysis, methemoglobinemia, and protein deficiency.

Given the complex metabolism of chloroquine and rasburicase and the causal hemolysis due to treatment of patients with G6PD deficiency, there is sufficient evidence for an implication of G6PD with hemolysis upon treatment with chloroquine and rasburicase, as also highlighted in the respective drug label issued by the FDA (McDonagh et al., 2012; Relling et al., 2014). G6PD is located in chromosome $\mathrm{X}$ with more than 100 different variants identified, within the Mediterranean and Middle Eastern populations and some of them more frequent among the Greek population, such as rs5030868 (Menounos et al., 2000).

Taken together, the genotyping of rs5030868 with the rapid PCR-ARMS (polymerase chain reaction amplification refractory mutation system) by $2 \mathrm{MoBiL}$ warrants consideration and field studies toward tailoring the chloroquine and rasburicase dose and safety monitoring (especially for the individuals with G6PD deficiency), and toward obtaining the benefits of chloroquine and rasburicase therapy in a safe and effective manner (Patrinos et al., 2020, unpublished data).

Future studies could implement the ability and accuracy of machine learning-based algorithms (such as random forest or decision tree) to assign a genotype score in PCR electrophoresis outputs as produced from the $2 \mathrm{MoBiL}$ method. For example, subsequent future studies could use the location of the detected gel bands, their distance to the corresponding column, their distance to the molecular ladder, and the ratio of their heights. Then, an ML-based (machine learning-based) algorithm could be trained to classify different PCR electrophoresis outputs according to their genotype. Such studies will further promote the robustness of $2 \mathrm{MoBiL}$ method combined with the accuracy of ML-based algorithms, thus advancing the field of personalized medicine and more precisely, for diagnostic purposes.

Moreover, since this is a first attempt in development efforts, further studies are needed in larger samples before clinical applications.

Overall, the 2MoBiL-based method, as described herein, is shown to be feasible. Apart from the potential use of these instruments in biodefence and educational purposes, as previously highlighted, they can be also used for drug dose adjustment purposes. Taking into consideration the compact size of $2 \mathrm{MoBiL}$, its feasibility, potentially low operational costs, and the high accuracy achieved in this study, future studies on its clinical validity and clinical utility, particularly in remote and sparsely populated areas or resource-limited global settings, are called for.

\section{Author Disclosure Statement}

The authors declare they have no competing financial interests.

\section{Funding Information}

This study was partly funded by a European Commission grant (H2020-668353; Ubiquitous Pharmacogenomics) to G.P.P.

\section{References}

Ahrberg CD, Ilic BR, Manz A, and Neužil P. (2016). Handheld real-time PCR device. Lab Chip 16, 586-592.

Brunham LR, Baker S, Mammen A, et al. (2018). Role of genetics in the prediction of statin-associated muscle symptoms and optimization of statin use and adherence. Cardiovasc Res 114, 1073-1081.

Jangam SR, Agarwal AK, Sur K, and Kelso DM. (2013). A point-of-care PCR test for HIV-1 detection in resourcelimitedsettings. Biosens Bioelectron 42, 69-75.

Khine H, Yuet WC, Adams-Huet B, and Ahmad Z. (2016). Statin-associated muscle symptoms and SLCO1B1 rs4149056 genotype in patients with familial hypercholesterolemia. Am Heart J 179, 1-9.

Koutsilieri S, Tzioufa F, Sismanoglou DC, et al. (2020). Unveiling the guidance heterogeneity for genome-informed drug treatment interventions among regulatory bodies and research consortia. Pharmacol Res 153, 104590.

Kurosaki Y, Magassouba N, Oloniniyi OK, et al. (2016). Development and evaluation of reverse transcription-loop- 
mediated isothermal amplification (RT-LAMP) assay coupled with a portable device for rapid diagnosis of Ebola virus disease in Guinea. PLoS Negl Trop Dis 10, e0004472.

Lim S, Nan H, Lee M, and Kang SH. (2014). Fast on-site diagnosis of influenza A virus by Palm PCR and portable capillary electrophoresis. J Chromatogr B Analyt Technol Biomed Life Sci 963, 134-139.

Macleod JA, Nemeth AC, Dicke WC, et al. (2016). Fast, sensitive point of care electrochemical molecular system for point mutation and select agent detection. Lab Chip 16, 2513-2520.

Marx V. (2015). PCR heads into the field. Nat Methods 12, 393-397.

McDonagh EM, Thorn CF, Bautista JM, et al. (2012). PharmGKB summary: Very important pharmacogene information for G6PD. Pharmacogenet Genomics 22, 219.

Meade T, Sleight P, Collins R, et al. (2008). SLCO1B1 variants and statin-induced myopathy-A genomewide study. N Engl J Med 359, 789-799.

Menounos P, Zervas C, Garinis G, et al. (2000). Molecular heterogeneity of the glucose-6-phosphate dehydrogenase deficiency in the Hellenic population. Hum Hered 50, 237-241.

Nair CB, Manjula J, Subramani PA, et al. (2016). Differential diagnosis of malaria on truelab Uno ${ }^{\circledR}$, a portable, real-time, MicroPCR device for point-of-care applications. PLoS One 11, e0146961.

Pasanen MK, Fredrikson H, Neuvonen PJ, and Niemi M. (2007). Different effects of SLCO1B1 polymorphism on the pharmacokinetics of atorvastatin and rosuvastatin. Clin Pharmacol Ther 82, 726-733.

Pasanen MK, Neuvonen M, Neuvonen PJ, and Niemi M. (2006). SLCO1B1 polymorphism markedly affects the pharmacokinetics of simvastatin acid. Pharmacogenet Genomics 16, 873-879.

Pirmohamed M. (2001). Pharmacogenetics and pharmacogenomics. Br J Clin Pharmacol 52, 345-347.

Relling MV, Mcdonagh EM, Chang T, et al. (2014). Clinical Pharmacogenetics Implementation Consortium (CPIC) guidelines for rasburicase therapy in the context of G6PD deficiency genotype. Clin Pharmacol Ther 96, 169-174.

Siamoglou S, Karamperis K, Mitropoulou C, and Patrinos GP. (2020). Costing methods as a means to measure the costs of pharmacogenomics testing. J Appl Lab Med 5, 1005-1016.

Siamoglou S, and Patrinos GP. (2019). 2MoBiL laboratory in educational activities in the field of molecular genetics and

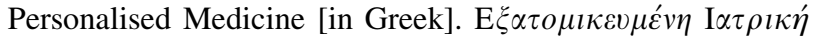
$1,17-25$.

Stroes ES, Thompson PD, Corsini A, et al. (2015). Statin-associated muscle symptoms: Impact on statin therapy-European Atherosclerosis Society consensus panel statement on assessment, aetiology and management. Eur Heart J 36, 1012-1022.

Teama S. (2018). DNA polymorphisms: DNA-based molecular markers and their application in medicine. Genetic Diversity and Disease Susceptibility, 25. DOI: 10.5772/intechopen .79517 .

Thorn CF, Klein TE, and Altman RB. (2013). PharmGKB: The pharmacogenomics knowledge base. Methods Mol Biol 1015, 311-320.

Tuteja S, and Rader DJ. (2018). SLCO1B1 and statin therapy: Getting the GIST of pharmacogenetic testing. Circ Genomic Precis Med 11, e002320.

Visser BJ, Meerveld-Gerrits J, Kroon D, et al. (2015). Assessing the quality of anti-malarial drugs from Gabonese pharmacies using the MiniLab ${ }^{\circledR}$ : A field study. Malar J 14, 1-13.

Wang H, Zhang X, Xu X, et al. (2017). A portable microfluidic platform for rapid molecular diagnostic testing of patients with myeloproliferative neoplasms. Sci Rep 7, 1-11.

Wilke RA, Ramsey LB, Johnson SG, et al. (2012). The clinical pharmacogenomics implementation consortium: CPIC guideline for SLCO1B1 and simvastatin-induced myopathy. Clin Pharmacol Ther 92, 112-117.

Zaky WI, Tomaino FR, Pilotte N, et al. (2018). Backpack PCR: A point-of-collection diagnostic platform for the rapid detection of Brugia parasites in mosquitoes. PLoS Negl Trop Dis 12, e0006962.

\section{Address correspondence to: Prof. George P. Patrinos Laboratory of Pharmacogenomics and Individualized Therapy Department of Pharmacy School of Health Sciences University of Patras University Campus, Rion Patras GR-26504 \\ Greece}

E-mail: gpatrinos@upatras.gr

\begin{tabular}{rl|} 
& Abbreviations Used \\
2MoBiL $=$ Mobile Molecular Biology Laboratory \\
$\mathrm{ADR}=$ adverse drug reaction \\
$\mathrm{AS}-\mathrm{PCR}=$ allele-specific polymerase chain reaction \\
$\mathrm{DNA}=$ deoxyribonucleic acid \\
$\mathrm{G} 6 \mathrm{PD}=$ glucose- 6 - phosphate dehydrogenase \\
$\mathrm{HIV}=$ human immunodeficiency viruses \\
$\mathrm{ML}=$ machine learning \\
$\mathrm{PCR}=$ polymerase chain reaction \\
PCR-ARMS $=$ polymerase chain reaction amplification \\
refractory mutation system \\
$\mathrm{SNP}=$ single-nucleotide polymorphism
\end{tabular}

